\title{
Distortions of Magnetotelluric Sounding Curves Due to a Slope
}

\author{
Geoexploration, 1988, 25, 229-244. \\ DOI 10.1016/0016-7142(88)90018-X
}

\author{
J.M. TRAVASSOS*1 and D. BEAMISH \\ British Geological Survey, Murchison House, West Mains Road, Edinburgh EH9 3LA (United \\ Kingdom)
}

\begin{abstract}
Travassos, J.M. and Beamish, D., 1988. Distortions of magnetotelluric sounding curves due to a slope. Geoexploration, 25: 229-244.
\end{abstract}

This study considers a particular case of 2D modeling, namely the topographic effect on MT data. A simple way to remove topographic effects is to incorporate surface elevations, or depressions, into the forward model. In cases where a $2 \mathrm{D}$ approximation is valid, any $2 \mathrm{D}$ algorithm may be used for the calculations. Such a scheme allows model results and observations to be compared along a profile. This approach, however, does not necessarily provide useful answers since the topography may, and in fact nearly always does, couple with the geoelectric structure and/or varies on a more rapid scale than the observations. In order to understand the effect of topography on MT data the degree and characteristics of the distortion introduced by a simple slope are considered. The slope model constitutes a general result with distortion characteristics that can be extended to other features such as a depression or a hill. The introduction of additional features such as a superimposed geoelectric structure would greatly complicate the problem due to electromagnetic coupling effects which would necessarily be a function of frequency. The single slope, or half-graben, is probably the simplest class of general near-surface distortions that affect MT data.

The period range used is from $0.02 \mathrm{~s}(50 \mathrm{~Hz})$ to $100 \mathrm{~s}(0.01 \mathrm{~Hz})$, i.e. both audio and magnetotelluric observations are considered. The elevation of the slope is fixed at a 'throw' of $1 \mathrm{~km}$ and slope angles range from $0^{\circ}$ to $90^{\circ}$. The spatial and frequency distortions due to the slope are considered in detail. At a particular location the distorting effect of the slope introduces a false gradient into an otherwise 1D sounding curve. The topographic distortion introduced is investigated by applying a $1 D$ inverse procedure to the obtained results. The way in which false layers may be introduced into the geoelectric section is illustrated. An analytical solution to a purely DC topographic problem is also considered. The analytical results are compared with the corresponding low frequency results obtained with the numerical models of the present study.

*1Also at: Departamento de Geofisica, CNPq - Observatorio Nacional, R. Gen. Bruce 586, 20921 Rio de Janeiro, RJ (Brazil) 
The presence of topography represents a complicating factor in the interpretation of magnetotelluric data. In general the problem is three-dimensional and frequency-dependent, although in some particular cases it may be approximated as two-dimensional. A recent review of topographical distortions of electromagnetic fields can be found elsewhere (Menvielle and Szarka, 1986). The objective of the present study is to analyse the frequency-dependent effect of a slope on an otherwise uniform half-space. This particular problem can be approximated by a two-dimensional model. In order to isolate the effect of the slope, the problem is kept as simple as possible by assigning a uniform conductivity to the earth.

The distortions introduced by topographic features have been studied for more than two decades. A number of different techniques have been used to model local topography. Plexiglass ridges have been used in analogue models (Faradzhev et al., 1972). Two-dimensional numerical approximations have been carried out using transmission system and network analogies (Ku et al., 1973; Mozley, 1982), finite elements (Wannamaker et al., 1986) and a modification of Rayleigh scattering theory (Jiracek, 1973) for modeling topographic features (Reddig and Jiracek, 1984). The simpler telluric case has been analysed with the aid of conformal transformation (Thayer, 1975; Harinarayana and Sarma, 1982). In addition it has been suggested that it may also be possible to normalize impedance tensor elements to compensate for the topographic distortions (Mozley, 1982).

The aim of this paper is to present some aspects which have been largely overlooked in the literature so far. In particular we illustrate the case of a 'topographic sounding curve' and how a uniform earth 1D interpretation is influenced by a $2 \mathrm{D}$ topographic feature. A comparison between a DC analytical approximation and our numerical results at long periods provides an independent check on the consistency of the 2D finite difference algorithm used here (Brewitt-Taylor and Weaver, 1976).

Only one model, i.e., a slope on a uniform earth, is considered here. Examples of particular situations where the topography is superinpused on an inhomogeneous earth can be found in the literature (Ku et al., 1973; Wanamaker et al., 1986). We consider the single slope model extremely useful for a qualitative discussion on the effect of the topography since for certain modes of induction the topographic response is most sensitive to local surface slopes. The single slope can be used to qualitatively assess the effect of a more complicated topography without the need for further model calculations. For instance the two flat parts of the slope model provide enough information to understand qualitatively the topographic responses outside a depression or a hill. This can be readily seen in results where slopes, depressions and hills are considered together (Wannamaker et al., 1986). Any discrepancies between the responses 
outside these features are certainly much less important than the combined effect of the topography and the (unknown) geoelectric structure.

\section{MODELING PROCEDURE}

For the modeling a finite difference algorithm (Brewitt-Taylor and Weaver, 1976) was used in calculating surface responses. A single basic grid was used for all the models considered. The $y$-axis is perpendicular to the strike direction and the $z$-axis points downwards. The grid is made of 51 nodes along the $y$ direction and 31 nodes along the $z$-direction. Since grid elements are rectangular, slopes are approximated by a series of steps. The height difference $(d)$ between lower and higher (horizontal) sides is kept constant, $d=1 \mathrm{~km}$, while allowing slope angles to vary from $10^{\circ}$ to $90^{\circ}$. The half-space below the slope is assumed to be of uniform resistivity, $\rho=100 \Omega . \mathrm{m}$.

In terms of model construction both overall grid size and the smallest distance between two grid points depend on the skin depths considered. Eight period values are considered here covering the range from $0.02 \mathrm{~s}$ to $100 \mathrm{~s}$. These provide skin depths varying from $0.71 \mathrm{~km}$ to $50.3 \mathrm{~km}$. Distances between grid points will depend on their proximity to the slope. The nearer to the slope a grid point, the smaller will be its distance to neighbouring grid points. The smallest grid size used was $0.2 \mathrm{~km}$. The whole grid is a square of dimensions $800 \times 800 \mathrm{~km}$. This is sufficient to ensure that any slope model is about 8 skin depths from the edges at $100 \mathrm{~s}$. Grid geometry was inspired by published guidelines (Doucet and Ngoc, 1984). The air is simulated by assigning a high resistivity value of $10^{19} \Omega . \mathrm{m}$. The horizontal electromagnetic field is calculated for both $\mathrm{E}$ and $\mathrm{H}$ polarizations through the grid. Apparent resistivity and phase are calculated along the surface. The finite difference algorithm produces stable results only outside the slope, thus restricting results to points situated outside the slope. Distances are thus measured from the left of the slope $y<0$ or from the right of it, $y>0$. Therefore when referring to results of a particular model in the text below, it will be assumed that the distance is measured from the nearest edge of the slope. Models are referred to by the angle of inclination $\theta$. Five models are considered here, $10^{\circ}, 20^{\circ}, 45^{\circ}, 60^{\circ}$ and $90^{\circ}$. Fig. 1 illustrates, in grid scale, the $45^{\circ}$ slope model used in the calculations. Note that the distances in this figure are grid distances and not distances from the slope.

Since the same grid was used for all periods, it is necessary to assess the quality of the results throughout the whole period range. For this purpose a half-space model with the surface at $z=0$ is used. Consider all grid points on the surface with grid distances $|y| \leq 100 \mathrm{~km}$. At each period the mean and standard deviation are obtained for the apparent resistivity and phase obtained along the surface. Both polarizations are considered together, giving a total of 90 values used in the calculation of both the mean and standard deviation. The half-space results show good numerical stability. The mean values for both 


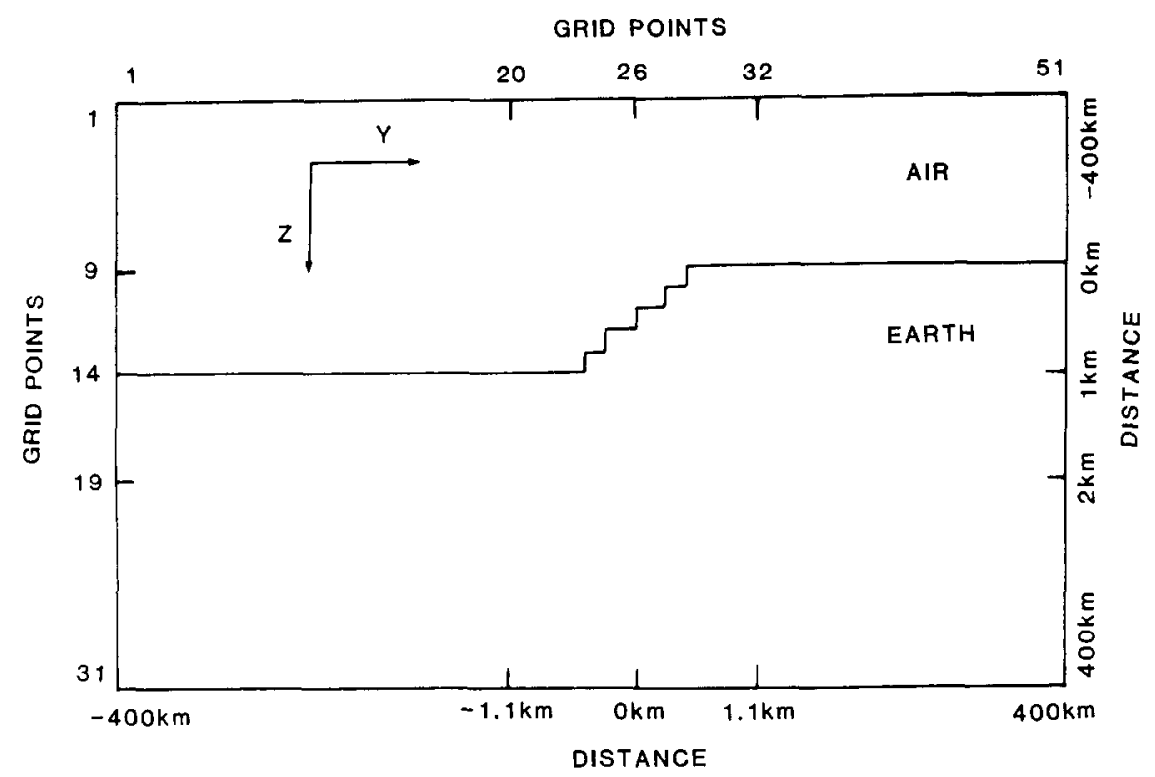

Fig, 1. Model, in grid scale, used for all numerical calculations. The centre point of the slope is at $0 \mathrm{~km}$.

apparent resistivity and phase agree with the theoretical values of $\rho=100 \Omega . \mathrm{m}$ and $\phi=45^{\circ}$, the biggest discrepancy being $0.3 \%$ for the apparent resistivity at $T=100 \mathrm{~s}$. The standard deviations are always less than $10 \%$. Restricting the calculation to points $|y| \leq 10 \mathrm{~km}$ makes the standard deviation drop to less than $7 \%$. Note that any slope model is always less than $6 \mathrm{~km}$ in length.

\section{SPATIAL DISTORTIONS DUE TO THE SLOPE}

We begin by analysing the distortions on the apparent resistivity and phase with distance for both E-polarization, $\rho_{\mathrm{E}}$ and $\phi_{\mathrm{E}}$, and $\mathrm{H}$-polarization, $\rho_{\mathrm{H}}$ and $\phi_{\mathrm{H}}$. This analysis is restricted to points $|y| \leq 8 \mathrm{~km}$ from the slope as distortions are always below $10 \%$ beyond this distance. For model calculations the degree of distortion is simply measured by the departure from the theoretical values, in other words of $\rho$ from $100 \Omega$.m and of $\phi$ from $45^{\circ}$.

Spatial distortions for the E-polarization case where induced currents flow parallel to the slope are presented in Fig. 2 for three selected periods. As can be seen both $\rho_{\mathrm{E}}$ and $\phi_{\mathrm{E}}$ are most distorted at the shortest period. As the period increases the amplitude of the distortion decreases but the distance where the effect can be detected increases. For periods $T \geq 1 \mathrm{~s}$ distortions are less than $10 \%$ and therefore are not presented. Also the distortions are stronger for points on the right of the slope, $y>0$ which correspond to a topographic high. At such points distortions of apparent resistivity can reach $70 \%$, although the effect is 


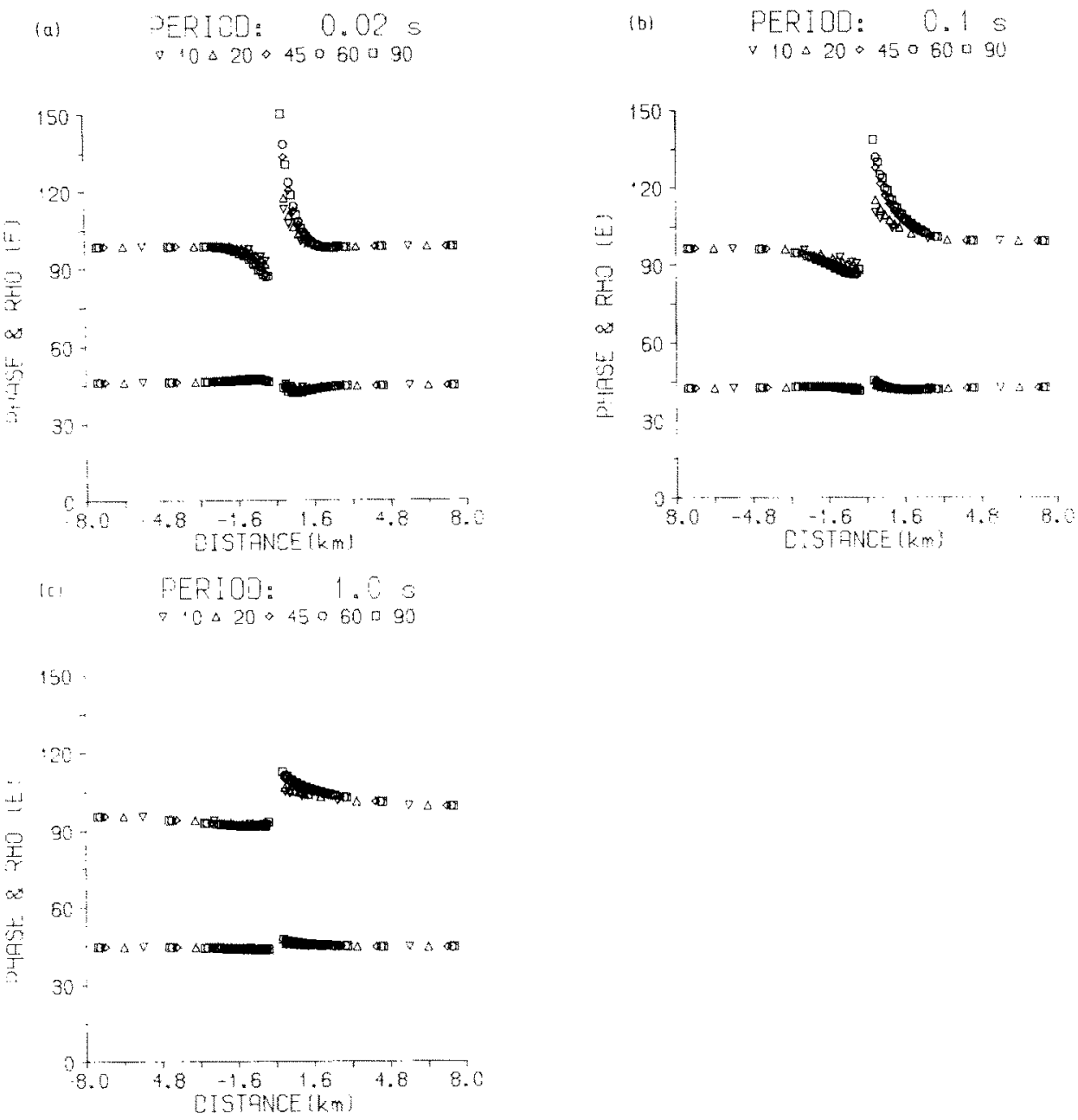

Fig. 2. E-polarization results. Apparent resistivity (RHO) and phase against distance from slope for three selected periods: (a) $0.02 \mathrm{~s}$; (b) $0.1 \mathrm{~s}$; and (c) $1 \mathrm{~s}$. Apparent resistivity in Ohm.m and phase in degrees throughout. In this and subsequent figures, results for the five slope models $\left(10^{\circ}\right.$, $20^{\circ}, 45^{\circ}, 60^{\circ}$, and $90^{\circ}$ ) are represented by the symbols shown. Note that grid geometry makes the relative distances from the slope differ for the five models.

very localized. At points $y<0$ distortions are limited to about $10 \%$. Phase distortions are small and follow the same pattern as the apparent resistivity, i.e. both follow the topography in the sense that slope distortion produces lower values on the lower side and higher values on the higher side. The E-polarization results shown in Fig. 2 are clearly frequency-dependent in character. This reflects to inductive nature of the distortion experienced in this mode. The maximum distortions occur when the skin depth in the medium is of the same order as the electromagnetic scale length of the topographic feature. Skin depths 
for the periods considered in Fig. 2 range from $0.71 \mathrm{~km}$ for $T=0.02 \mathrm{~s}$ to 11.2 $\mathrm{km}$ for $T=5 \mathrm{~s}$. The results also demonstrate that the level of distortion increases with slope angle.

It is well known that the H-polarization case, when induced currents are perpendicular to the slope, is more affected by topography than the E-polarization case. Fig. 3 shows both $\rho_{\mathrm{H}}$ and $\phi_{\mathrm{H}}$ for three selected periods. The longer the period, the more affected is the apparent resistivity, while the phase is distorted above $10 \%$ only at points $y>0$ for periods $T \leq 1 \mathrm{~s}$. Moreover the phase is more affected at shorter periods, as in the E-polarization case, but clearly $\rho_{\mathbf{H}}$

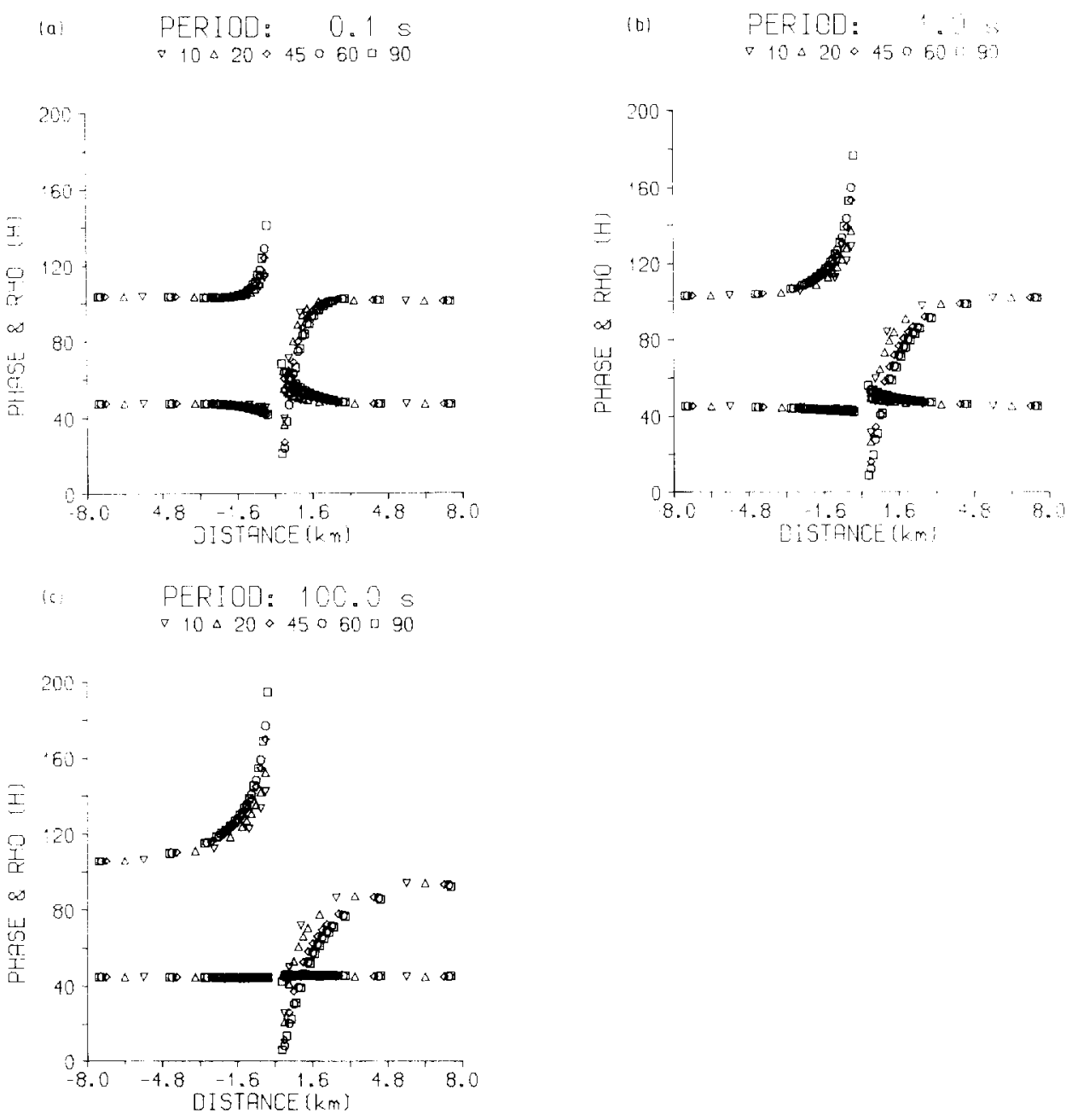

Fig. 3. H-polarization results. Apparent resistivity ( $\mathrm{RHO}$ ) and phase against distance from slope for three selected periods: (a) $0.1 \mathrm{~s}$; (b) $1.0 \mathrm{~s}$; and (c) $100.0 \mathrm{~s}$. The five slope models are represented by the symbols shown. 
displays the reverse behaviour. Values of $\rho_{\mathrm{H}}$ can be distorted in excess of $100 \%$ on both sides of the slope.

It is apparent from the results of Fig. 3 that substantial distortions of $\rho_{\mathrm{H}}$ persist across a large bandwidth, the degree of distortion increasing with increasing period. The variation with slope angle is only a marginal effect. For the same slope elevation, distortions are similar in magnitude, but not in sign, on the topographic high and low. The general distortion features obtained in our study, for both polarization modes, are compatible with previous model results (Ku et al., 1973: Reddig and Jiracek, 1984; Wannamaker et al., 1986) although numerical algorithms and models considered differ in all four cases.

\section{DISTORTIONS OF SOUNDING CURVES AT PARTICULAR SITES}

We now investigate how sounding curves are distorted at particular sites. Six sites are selected: $|y|=0.4,1$, and $2 \mathrm{~km}$. The least distorted E-polarization case is considered first. As indicated in Fig. 2, only sites near the slope are significantly affected, so the analysis is restricted to distances $|y| \leq 1 \mathrm{~km}$. The two extreme sites $|y|=2 \mathrm{~km}$ show distortions which are below $10 \%$ and thus may be neglected. Fig. 4 shows the sounding curves for the four nearer sites. For these four sites distortions can reach $15 \%$ on the lower side and $50 \%$ on the higher side. It is important to note that these distortions are only significant for periods shorter than $1 \mathrm{~s}$ for both topographic high and low. In the context of the scale of the topographic feature here considered, we conclude that the topography would have only a marginal distorting effect for low frequency, i.e., MT, E-polarization results.

H-polarization-derived sounding curves are strongly distorted at all 6 sites as shown in Fig. 5. Unlike the E-polarization case, the phase is also significantly distorted in the immediate vicinity of the slope on the topographic high (Fig. 5d). On the lower side, phases suffer very little distortion but apparent resistivities suffer distortions which are of the same order as the ones experienced on the higher side and reach $100 \%$. Note that the period range considered here does not extend sufficiently into the longer periods to allow the apparent resistivity curves to return to the undistorted levels.

As can be inferred from the last two figures the effect of the slope is essentially to produce an artificial two-layer sounding curve at the most distorted locations. In order to investigate such effects, the sounding curves are inverted at two sites, $|y|=1 \mathrm{~km}$. For this inversion a 1D linearized inversion scheme (Jupp and Vozoff, 1975) is employed. Both apparent resistivity and phase are jointly inverted and all inversions have the same two-layer starting model with $\rho_{1}=\rho_{2}=100 \Omega . \mathrm{m}$ and $h=10 \mathrm{~km}$ for the depth to the second layer, or half-space.

Inversions of the E-polarization data show very little deviation from the true structure. For the purposes of this investigation the 'true' structure would be returned as $\rho_{1}=\rho_{2}=100 \Omega . \mathrm{m}$ and $h=10 \mathrm{~km}$. For $|y|=1 \mathrm{~km}$ inversions are vir- 

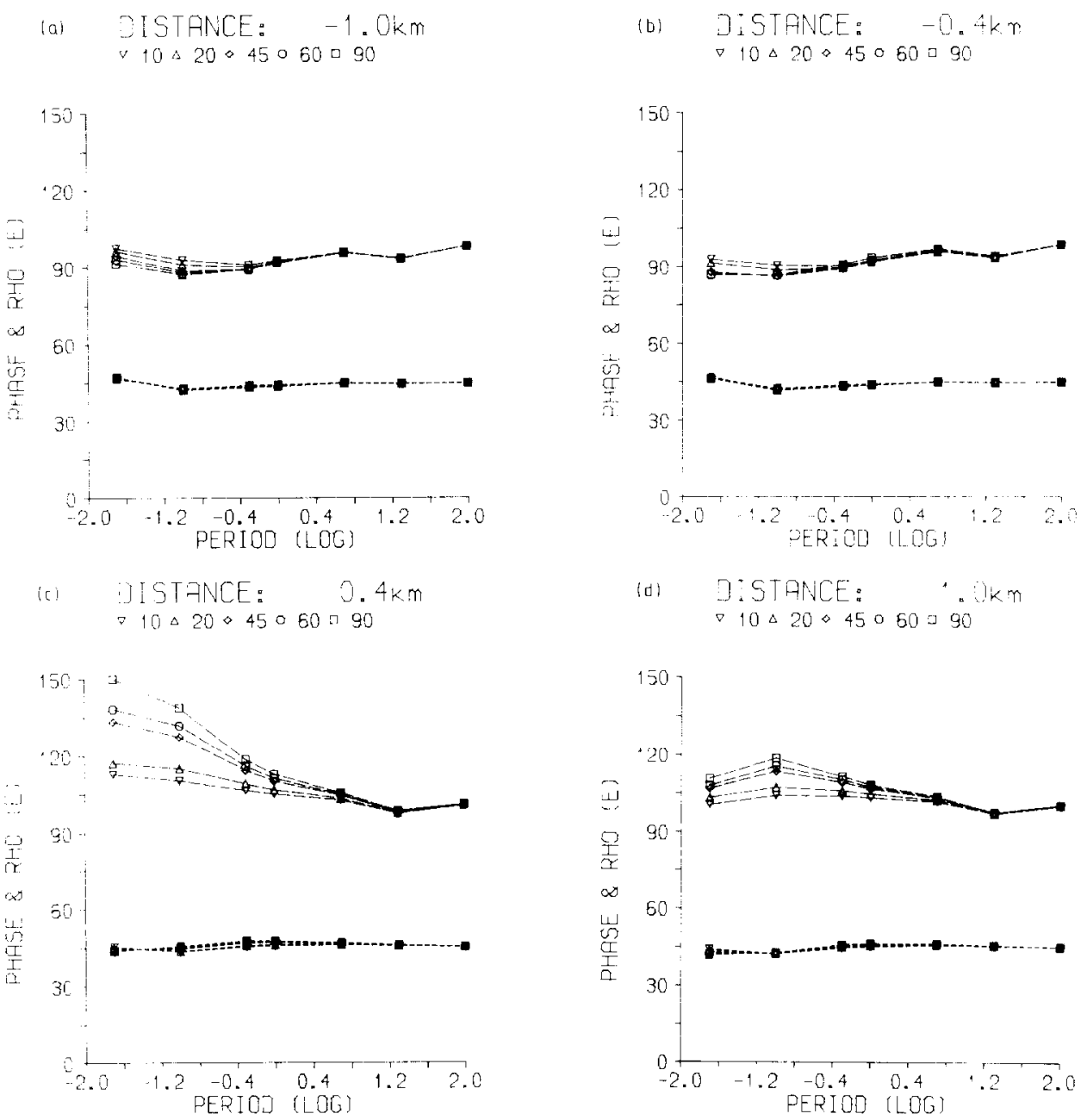

Fig. 4. E-polarization results. Sounding curves, apparent resistivity (RHO) and phase, at four selected locations. Location distances from the slope are: (a) $-1.0 \mathrm{~km}$; (b) $-0.4 \mathrm{~km}$; (c) $0.4 \mathrm{~km}$; and (d) $1.0 \mathrm{~km}$. The five slope models are represented by the symbols shown.

tually unaffected by the presence of the slope. However, inversions of the $\mathrm{H}$ polarization data are affected by the slope. The data we consider are shown in Fig. 5, b and e. The topographic distortions produce an apparent (false) thin layer over the half-space; the greater the slope angle, the thinner this first layer becomes. Fig. 6 shows the depth to the half-space against slope angle on the

Fig. 5. H-polarization results. Sounding curves, apparent resistivity ( $\mathrm{RHO}$ ) and phase, at six selected locations. Location distances from the slope are: (a) $-2.0 \mathrm{~km} ;(\mathrm{b})-1.0 \mathrm{~km}$; (c) -0.4 $\mathrm{km}$; (d) $0.4 \mathrm{~km}$; (e) $1.0 \mathrm{~km}$; and (e) $2.0 \mathrm{~km}$. The five slope models are represented by the symbols shown. 

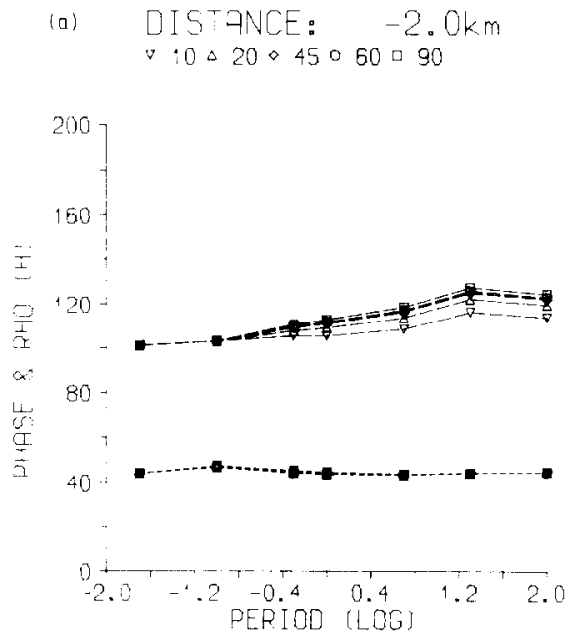

(c) SISTANCE: $\quad-0.4 \mathrm{~km}$
$\nabla 10 \triangle 20045060090$
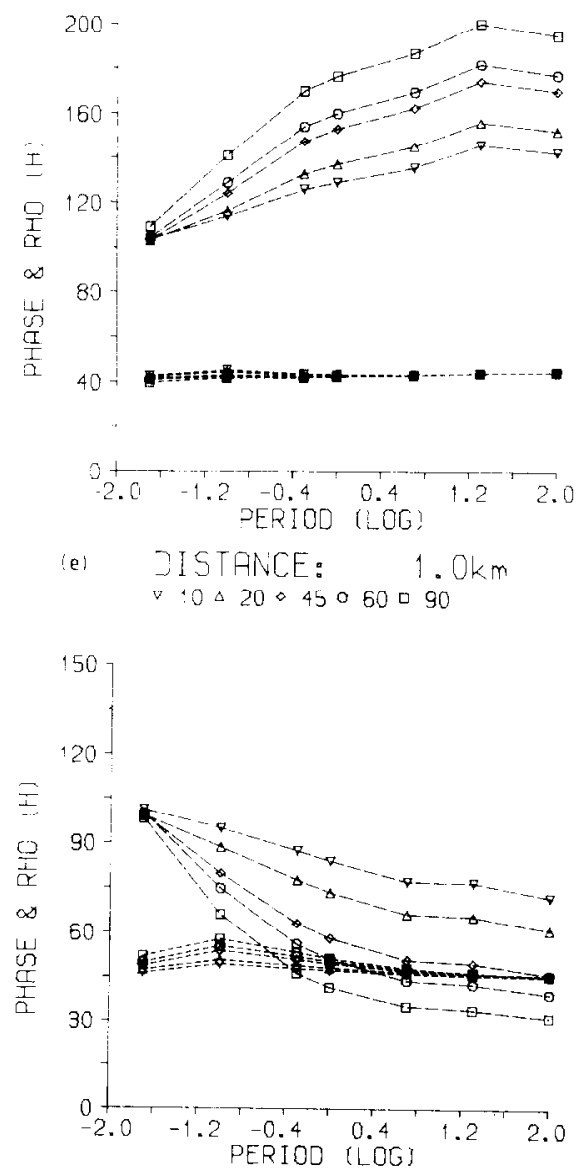

(b) BISTANCE: $-1.0 \mathrm{~km}$ $\checkmark 10 \Delta 20 \otimes 45060 \square 90$

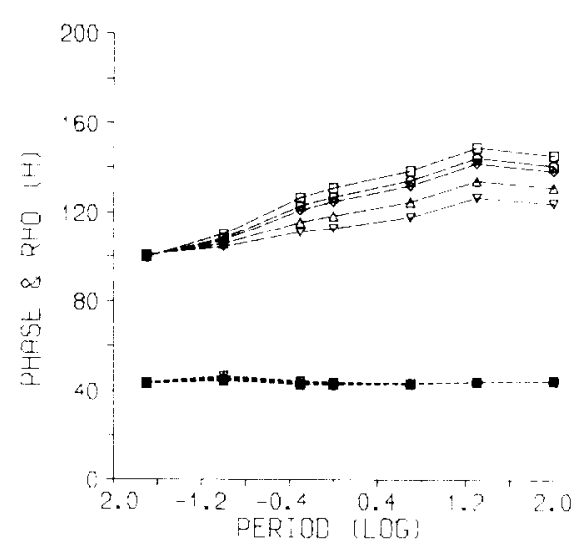

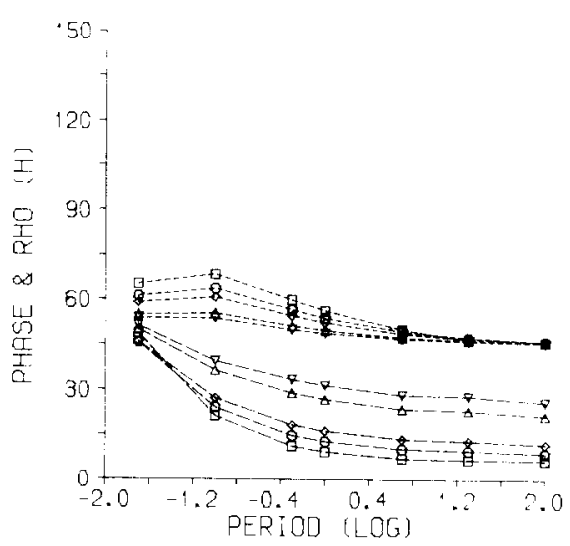
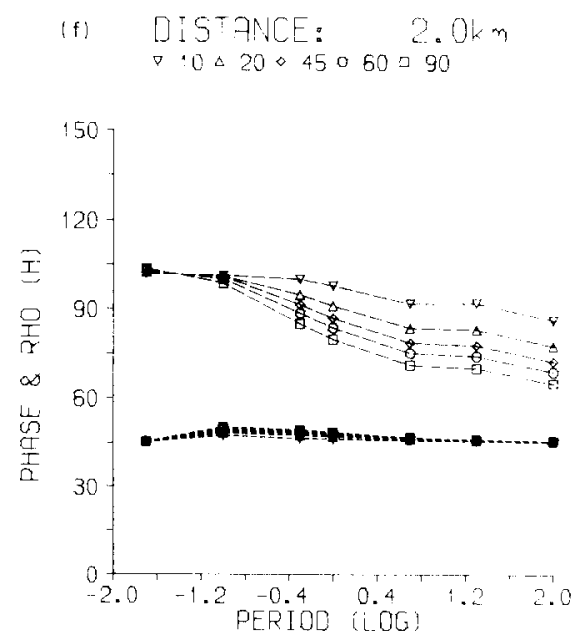


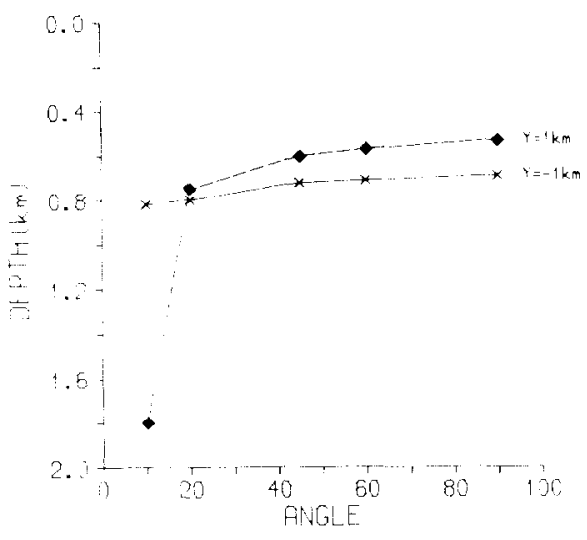

Fig. 6. Results of 1D inversion. H-polarization. Depth to half-space for the five slope models at two locations, $y=-1 \mathrm{~km}$ (solid line, cross symbols) and $y=1 \mathrm{~km}$ (dash line, diamonds).
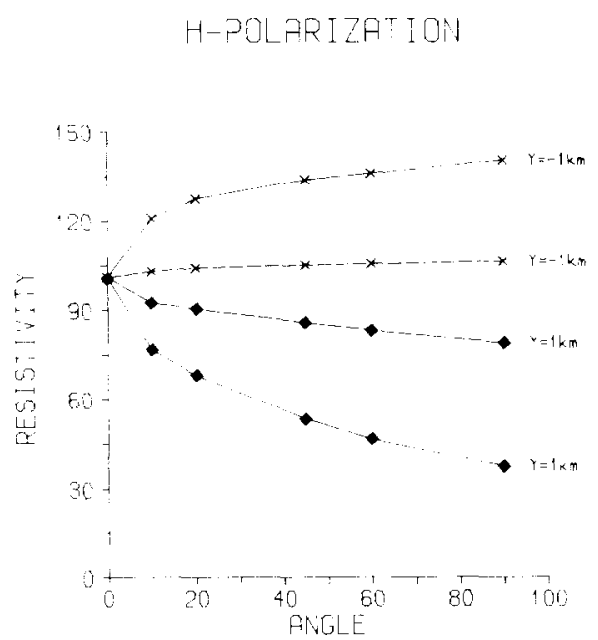

Fig. 7. Results of 1D inversion. H-polarization. Resistivities of first layer (solid lines) and halfspace (dash lines) for the five slope models at two locations, $y=-1 \mathrm{~km}$ (upper two curves, cross symbols) and $y=1 \mathrm{~km}$ (lower two curves, diamonds).

topographic low $(y=-1 \mathrm{~km})$ and on the topographic high $(y=1 \mathrm{~km})$. The resistivity of the first layer differs little from the true value, less than $5 \%$ on the lower side and up to $23 \%$ on the higher side. Of more interest is the resistivity of the half-space. As shown in Fig. 7, the resistivity values for the half-space 
can vary by $63 \%$ from the true value. This is important since it suggests that even minor topographic slopes can provide completely false geoelectric sections for the H-polarization case.

It is common practice to use rotationally invariant quantities constructed from combinations of the impedance tensor elements for $1 \mathrm{D}$ modeling. It is therefore worthwhile repeating the last investigation using the effective response given by:

$Z_{\text {eff }}=\left(Z_{x x} Z_{y y}-Z_{x y} Z_{y x}\right)^{1 / 2}$

(Berdichevsky and Dmitriev, 1976). For our 2D model the impedance elements $Z_{x x}$ and $Z_{y y}$ are true zeros and thus the effective response is the geometric mean of the E- and H-polarization results. The same two symmetrical points $y=1 \mathrm{~km}$ are used for this investigation. Inversions obtained using the invariant are less affected than for the H-polarization case. Fig. 8 shows that at $y=-1 \mathrm{~km}$, resistivity values for the two layers differ by less than $15 \%$ of the true value. Nevertheless at $y=1 \mathrm{~km}$ the resistivity of the half-space is between $12 \%$ and $36 \%$ below the correct value.

This same information can be presented in terms of a $2 \mathrm{D}$ section for a particular slope. Fig. 9 shows the half-space resistivities obtained by $1 \mathrm{D}$ inversion of the effective response $Z_{\text {eff }}$ at eight sites for a slope of $45^{\circ}$. 'The inversion results are assembled together to give an idea of the false interpretations which may be introduced by the topography. The resistivity values and depth to the half-space are shown.

As described above, the $\mathrm{H}$-polarization case is more affected by the presence of the slope. Distortions have a larger spatial wavelength and the level of dis-

$$
\text { INVARIANT }
$$

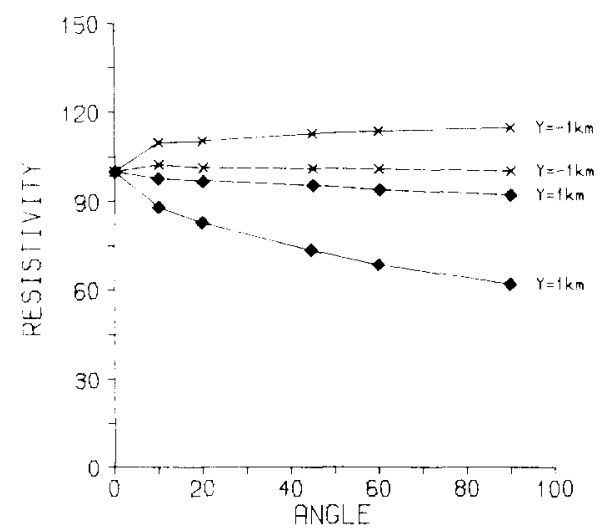

Fig. 8. Results of 1D inversion. Invariant (effective) response, for comparison with H-polarization response of previous figure. Same symbols apply. 


\section{DEGREES}

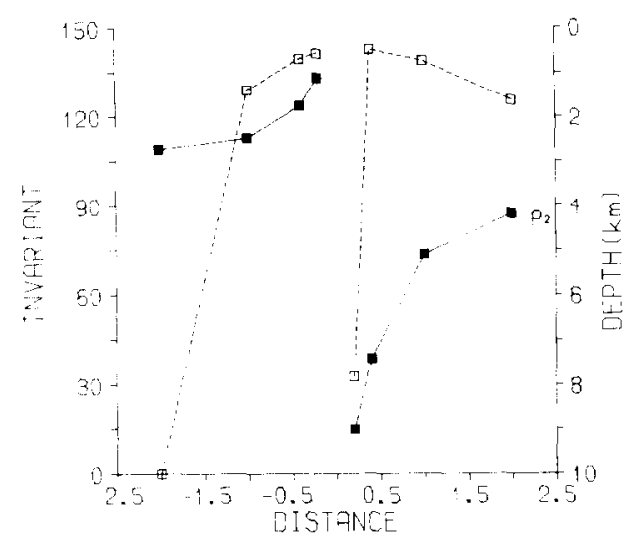

Fig. 9. Results of $1 \mathrm{D}$ inversion across the slope. Invariant (effective) response for a slope angle of $45^{\circ}$. Distance from the slope in $\mathrm{km}$. Resistivity of the half-space shown as a solid line (solid squares). Depth to the half-space shown as a dash line (open squares).

tortion increases with period. This is a direct consequence of the way current lines adjust themselves beneath the slope surface. In the H-polarization case currents flow perpendicular to the strike, the current lines are brought together beneath the corner which marks the transition from the topographic low and are pulled apart beneath the corner at the transition to the topographic high ( see for example Fox et al., 1980). For the E-polarization case the same mechanism of focusing and dispersion of current lines applies but as currents flow parallel to strike the effect is very localized. An infinite depth to the basement has been simulated with the $2 \mathrm{D}$ model used here. This has been done to isolate the purely topographic effect from additional coupling effects which would be introduced by additional structure in the forward model. One can, however, regard our $\mathrm{H}$-polarization results as an upper distortion limit to a finite depth to basement case (Faradzhev et al., 1972; Thayer, 1975).

Considering only the apparent resistivity for the $\mathrm{H}$ polarization case, the longer the period the more affected the sounding curves are, and distortions are more drastic for the $90^{\circ}$ model. These facts point to an upper limit to the distortions one should expect from an earth of uniform resistivity. However, the general case when the presence of subsurface structure may couple with the topography provides a very difficult problem. These facts direct the solution to be either a simple and generic one or to be a highly complex one, restricted to a particular situation. The point to be addressed in the generic case is, having the choice, how far from a topographic structure one should perform measurements in order to keep the topographic distortions to a minimum, say $10 \%$ or less? Equally important is to have a simple way of evaluating the dis- 
tortions since the actual field problem may be so complicated that it is not viable to solve it. Note also that the use of techniques like the ones for removing local superficial conductivity (Larsen, 1977), need such an extensive knowledge of the topography (Mozley, 1982), that it is hardly of any practical use when applied to topographic distortions.

\section{A COMPARISON WITH AN ANALYTICAL APPROXIMATION}

A simple analytical solution for telluric problems can be achieved using the Schwartz-Christoffel transform (Thayer, 1975; Harinarayana and Sarma, 1982 ). In this case telluric currents flow perpendicular to the strike between the surface and a perfect insulating basement. This solution, of course, is only valid for DC currents. Here it is shown that the telluric solution is a reasonable approximation to the limiting (low frequency) case in magnetotellurics.

The analytical model is a slope of height $d$ and depth to the basement $h$. In order to conform with the previous models it is assumed $d \ll h$; it is sufficient to set $h=100, d=1$. In what follows the results for the $\mathrm{H}$-polarization case are used for our longest period $T=100 \mathrm{~s}$. The analytical results were calculated by T. Harinarayana (pers. comm., 1985) for all the angles used in the present study. Results for the left of the slope models have already been published (Harinarayana and Sarma, 1982). The electric fields are assumed parallel to the $y$-direction and the same sign convention is used, i.e. $y<0$ on the left and $y>0$ on the right of the slope.

Let $E(y->\infty)=E_{\mathrm{h}}$ and $E(y->-\infty)=E_{1}$ be the limiting analytical values. It is known that (Thayer, 1975):

$E_{\mathrm{h}} / E_{\mathrm{l}}=h /(h+d)$

but as $d \ll h, E_{\mathrm{h}}=E_{1}=E_{\infty}$. The analytical field values are normalized as:

$\epsilon=E / E_{\infty}$

For our numerical models the field at infinity is approximated by an average of field values at points at a sufficient distance to be considered unaffected by the presence of the slope, $\bar{E}$. Thus a numerical counterpart of definition $l$ is given by:

$\epsilon^{\prime}=E^{\prime} / \bar{E}$

where $E^{\prime}$ and $\bar{E}$ are the computed values of our models. The comparison between $\epsilon$ and $\epsilon^{\prime}$ is done assuming that they express similar quantities and it is limited to $y_{\leq} \leq 5 \mathrm{~km}$ where distortions are $\geq 10 \%$.

Fig. 10 shows the values for $\epsilon$ and $\epsilon^{\prime}$ at points on the lower side, $y<0$. The two quantities agree reasonably well for all slope models except $10^{\circ}$, where some limited disagreement is apparent. Fig. 11 shows the same two quantities now on the higher side $y>0$. The two quantities now agree well only for $90^{\circ}$ 

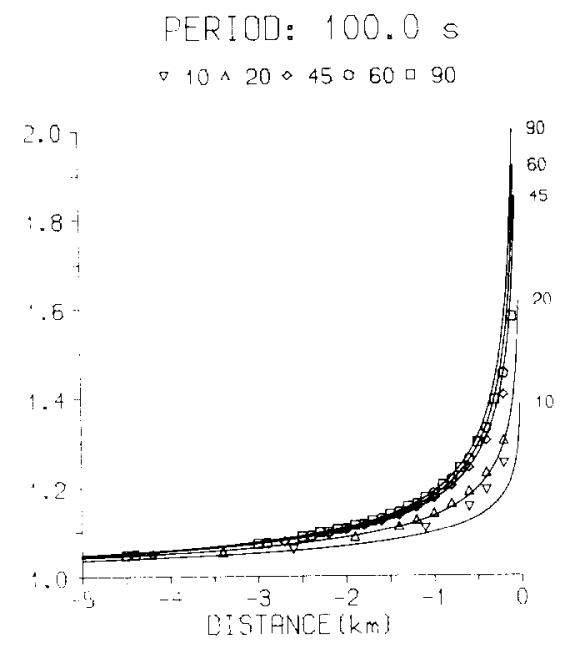

Fig. 10. Comparison between analytical and numerical model results (as described in the text) on the lower side of the slope. Normalised analytical results $(\epsilon$, solid lines) and numerical counterparts ( $c^{\prime}$, symbols shown) are plotted against distances for the five slope models.

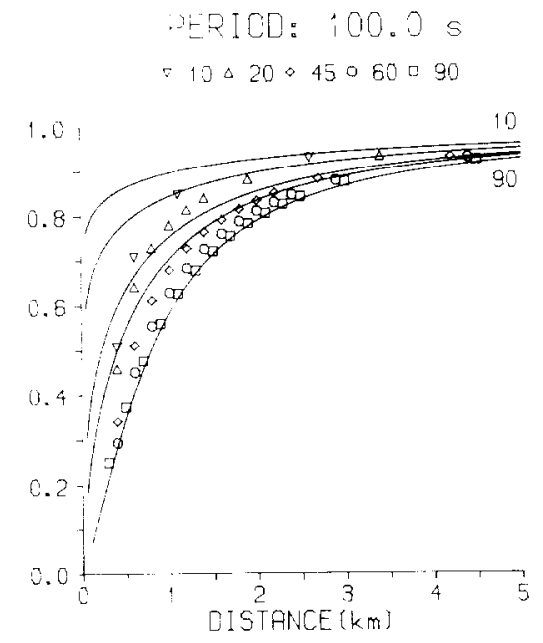

Fig. 11. As per Fig. 10 but on the upper side of the slope.

although $\epsilon$ and $\epsilon^{\prime}$ tend to agree away from the slope. Discrepancies between $\epsilon$ and $\epsilon^{\prime}$ are always less $10 \%$ at points $y>1 \mathrm{~km}$. Analytical results tend to be more symmetrical than the numerical results for small angles. As both analytical and numerical results agree well for the $90^{\circ}$ model for which distortions are more drastic, it is possible to conclude that the analytical approximation agrees well with the upper limit of the topographic distortions. An interesting by-product of this investigation is that the comparison between $\epsilon$ and $\epsilon^{\prime}$ provides an independent way of comparing the finite differences algorithm used 
in this work (Brewitt-Taylor and Weaver, 1976) with analytical results. Although the analytical results give only an approximation, both sets of results are in reasonable agreement.

\section{CONCLUSIONS}

A theoretical 2D model study has been undertaken of the topographic distortion on MT parameters introduced by a simple slope. In order to isolate the effect of the slope the problem has been kept as simple as possible by assigning a uniform conductivity to the earth. The slope, or half-graben, was modeled as having a fixed fault-throw of $1 \mathrm{~km}$ and inclinations were varied from $10^{\circ}$ to $90^{\circ}$. The electromagnetic scale length of the feature varies between $1 \mathrm{~km}\left(90^{\circ}\right.$ slope) and $6 \mathrm{~km}\left(10^{\circ}\right.$ slope $)$. The distortions introduced by this 'fixed' feature were analysed across a frequency range from $50 \mathrm{~Hz}$ to $0.01 \mathrm{~Hz}$, for both $\mathrm{E}$ - and $\mathrm{H}$-polarization modes. The skin depths of the inducing fields range from 0.71 to $50 \mathrm{~km}$.

Inductive, i.e. frequency-dependent, distortions occur for the E-polarization mode. The effect is very localised and appears to maximise when the skin depth is of the same order as the electromagnetric scale length of the topography. Distortions in both apparent resistivity and phase follow the topography in that values decrease on the low side and increase on the high side of the feature. Our investigations on the $1 \mathrm{D}$ inversion of the E-polarization results suggest that distortion effects can be neglected at distances greater than $1 \mathrm{~km}$ from the topographic margins.

Topographic distortions arising in the $\mathrm{H}$-polarization mode are much more severe. Unlike the E-polarization mode, distortions are significant on both sides of the slope and can reach $100 \%$. The degree of distortion increases with increasing period. The effect of the slope in this mode is to produce an artificial two-layer sounding curve at the most distorted locations. Such a distortion may be interpreted as a false first layer of thickness similar to the elevation of the feature. Within $1 \mathrm{~km}$ of the topographic margins, interpreted resistivities may vary by as much as $44 \%$ on the low and $63 \%$ on the high side. $1 \mathrm{D}$ inversions which use a combined, invariant, response are also influenced by the topography although not as much as in the H-polarization mode.

Distortions for both polarization modes are found to be related to slope angle, i.e., greater angles produce stronger distortions. The effect is, however, marginal when compared to the stronger influence of distance from the slope margin. The combined results single out the distortions introduced by the $\mathrm{H}$ polarization mode for a slope of $90^{\circ}$ as producing the maximum topographic distortion. This limiting case at a period of $100 \mathrm{~s}$ has been compared with an analytical approximation which is strictly valid only for DC currents. Both numerical and analytical results agree well, although the latter tend to underestimate the topographic effect if other angles are considered. 


\section{ACKNOWLEDGEMENTS}

The authors gratefully acknowledge the analytical data set used in this work provided by T. Harinarayana. One of the authors (JMT) acknowledges the award of a research studentship from the Brazilian Research Council (CNPq). This paper is published with the permission of the Director, British Geological Survey (NERC).

\section{REFERENCES}

Berdichevsky, M.N. and Dimitriev, V.I., 1976. Distortion of magnetic and electrical fields by surface lateral inhomogeneities. Acta Geod., Geophys. Mont. Acad. Sci. Hung., 11 (3-4): 447483.

Brewitt-Taylor, C.R. and Weaver, J.T., 1976. On the finite difference solution of two-dimensional induction problems. Geophys. J. R. Astron. Soc., 47: 375-396.

Doucet, D. and Ngoc, P.V., 1984. Généralisation et optimalisation de la méthode des différences finies pour la modélisation en magnetotellurique (MT). Geophys. Prospect., 32: 292-316.

Farudzhev, A.S., Kakhramanov, K.K., Sarkisov, G.A. and Khalilova, N.E., 1972. On effect of terrain on results of magnetotelluric soundings (MTS) and profiling (MTP). Izw., Earth Phys., 5: $92-94$.

Fox, R.C., Hohmann, G.W., Killpack, T.J. and Rijo, L., 1980. Topographic effects in resistivity and induced-polarization surveys. Geophysics, 45: 75-93.

Harinarayana, T. and Sarma, S.V.S., 1982. Topographic effects on telluric field measurements. PAGEOPH, 120: 778-783.

Jiracek, G.R.. 1973. Numerical comparisons of a modified Rayleigh approach with other rough surface EM scattering solutions. IEEE Trans. Ant. Propagation, AP21, 3: 393-396.

Jupp, D.L.B. and Vozoff, K., 1975. Stable iterative methods for the inversion of geophysical data. Geophys. J.R. Astron. Soc., 42: 957-976.

Ku, C.C., Hsieh, M.S. and Lim, S.H., 1973. The topographic effect in electromagnetic fields. Can. J. Earth Sci., 10:645 650.

Larsen, J.C., 1977. Removal of local surface conductivity effects from low frequency mantle response curves. Acta Geod., Geophys. Mont. Acad. Sci. Hung., 12: 183-186.

Menvielle, M. and Szarka, L., 1986. Distortions of electromagnetic fields: topographic and manmade. Review paper at the 8th IAGA Workshop on Electromagnetic Induction, Neuchatel, 1986. To appear in: Phys. Earth Planet. Inter.

Mozley, E.C. 1982. An Investigation of the Conductivity Distribution in the Vicinity of a Cascade Volcano. PhD Dissertation, University of California, Berkeley, Calif.

Reddig, R.P. and Jiracek, G.R., 1984. Topographic modelling and correction in magnetotellurics. Extended abstract to the 54th SFG, Atlanta, USA.

Thayer, R.E. 1975. Topographic distortion of telluric currents: a simple calculation. Geophysics, 40 (1): 91-95.

Wannamaker, P.E., Stodt, J.A. and Rijo, L., 1986. Two-dimensional topographic responses in magnetotellurics modelled using finite elements. Geophysics, 51 (11): 2131-2144. 